\title{
Verankerung der Ethikberatung im klinischen Alltag
}

\section{Jean Martin}

Dr. med., Mitglied der Redaktion und ehemaliges Mitglied der Nationalen Ethikkommission

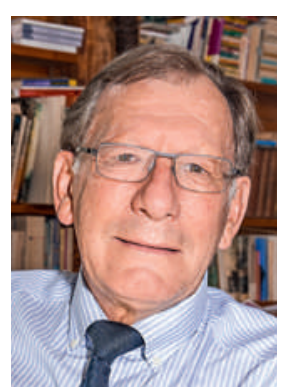

Die Schweizerische Akademie der Medizinischen Wissenschaften hat im Jahr 2012 Empfehlungen zur ethischen Unterstützung in der Medizin ausgesprochen. Am 21. April 2016 veranstaltete sie in Bern ein Symposium mit etwa einhundert Fachleuten, die Herausforderungen und Perspektiven für die Zukunft aufzeigten. In diesem Kontext muss jedoch zunächst (wie fast immer!) darauf hingewiesen werden, dass es keine Patentlösung dafür gibt, wie Ethikberatung richtigerweise im Spital, im Alten- und Pflegeheim oder in der ambulanten Praxis stattfinden sollte. Dazu die Ethikbeauftragte der Klinik St. Anna in Luzern: "Wichtig ist nicht, wie eine schwierige Situation analysiert wird, sondern dass sie überhaupt analysiert wird.» Diese Aussage mag erstaunen, aber Fakt ist, dass verschiedene Wege möglich sind. In einem ersten Schritt müssen die zuständigen Gremien dieser Aufgabe den notwendigen Raum geben - und die erforderlichen Mittel. Einer der Redner führte an, dass Skandale institutionellerseits ethisches Handeln bewirken können, doch es ist natürlich vorzuziehen, nicht ein solches Vorkommnis abzuwarten! Thematisiert wurde die mögliche Professionalisierung der Ethikberatung. Auf kurze Sicht scheint die Lösung nicht darin zu bestehen, nach einer streng geregelten, singulären Schulung Ausschau zu halten. In der Tat kann die Ethikberatung von Ärzten, Pflegekräften, Philosophen, Psychologen oder auch Juristen angeboten werden. Die Beratungsanbieter müssen über relevante und nachhaltige - auch theoretische - Erfahrung verfügen und sich in diesem Bereich engagiert haben. Auch sollten sie interdisziplinäre Reflexionen und Debatten führen können.

Professor Bert Molewijk (Amsterdam) referierte über die «Moral Case Deliberation», einen kontextuellen, pragmatischen klinischen Ansatz, verankert in der Erfahrung der Gesundheitsbetreuer, die ihre Befragungen in einen strukturierten Dialog bringen, unterstützt durch einen Ethiker [1]. «Die ethische Unterstützung ist kein Selbstzweck», erklärte Molewijk und stellte nochmals klar, dass es sich um eine Rolle/Leistung im Dienste derjenigen handle, die an vorderster Front sind. Er unterstrich ferner, wie wichtig es sei, bei jenen, die eine Beratung einfordern, ein Gefühl der Miteignerschaft und der Mitverantwortung im ethi- schen Handeln und den daraus gezogenen Schlussfolgerungen zu stimulieren. Die Ethikkommissionen und -berater diskutieren Werte, Rechte und Interessen, die in den jeweiligen Situationen zum Tragen kommen, und skizzieren mögliche Wege/Handlungen (gegebenenfalls den "weniger schlechten» Weg). Vor allem aber auch Unstimmigkeiten, die im Team selbst aufkommen. Sie formulieren Analysen oder Empfehlungen. In der Regel aber bleibt die Verantwortung der praktischen Entscheidung am Krankenbett in den Händen der zuständigen Ärzte und des Pflegeteams. Ethische Positionen werden nicht aufgezwungen, es wird darüber diskutiert und ein Konsens gesucht.

Die Leiterin des Ethikteams eines Universitätsspitals stellte fest, dass Chefärzte öfter komplexe Situationen vorlegen als rangjüngere Kollegen. Fürchten letztere vielleicht, dass ihre Fragen als Unzulänglichkeit gewertet werden könnten? Oft sollte ein «Ich weiss nicht» doch vielmehr als eine Art Verantwortung, ja selbst Mut gewertet werden. Es braucht viel Mut, um die Aufmerksamkeit des Chefs auf ethisch fragwürdige Aspekte in seiner Abteilung zu lenken ... Jemand aus einem deontologisch eher traditionell ausgerichteten Umfeld fügte an, dass Unstimmigkeiten zwischen Ärzten und offenerem Pflegepersonal häufig anzutreffen seien. Ellen Fox, amerikanische Expertin an der Clarkson University, sprach von "psychological safety»: Fruchtbare Diskussionen im Team ergeben sich nur in psychologischer Sicherheit, d.h. in einem vertrauensvollen Rahmen, unterstützt durch Zuhören und gegenseitigen Respekt. Dies ist von grundlegender Bedeutung.

Ziel ist eine echte Ethikkultur in der gesamten Gesundheitsbetreuung (beachtenswert dazu eine interessante, jüngst erschienene Veröffentlichung aus der Romandie [2]). Als einfacher Leitfaden wurde auch an den Satz des Genfer Ethikers Eric Fuchs erinnert, der da sagte, Ziel der Ethik sei es, zu schauen, «wie man handeln soll, um gerecht zu handeln».

\section{Literatur}

1 Molewijk B, Abma T, Stolper M, Widdershoven G. Teaching ethics in the clinic. The theory and practice of moral case deliberation. Journal Med. Ethics. 2008;34:120-4.

2 Corbaz $\mathrm{P}$, Quinche F. Ethiques pour les soins à domicile. Genève: Editions Médecine et Hygiène; 2015. 